\title{
TIPOLOGÍA AXIOMÁTICA DE LAS TEORÍAS EMPIRICAS
}

C. ULISES MOULINES

Universidad de Bielefeld

Presentar una teoría científica en forma axiomática es muy útil, no sólo por razones de economía intelectual, sino para cumplir una de las tareas básicas de la filosofía de la ciencia: identificar teorías. Esto vale, a mi parecer, no sólo para las teorías puramente matemáticas, cuya axiomatización es apreciada por casi todo el mundo, sino también para las teorías con aplicación empírica. En un trabajo reciente he aducido algunos argumentos en favor de esta tesis. ${ }^{1}$ Aquí no voy a repetirlos. La exposición que sigue va dirigida primordialmente a quienes están persuadidos de antemano de la conveniencia de axiomatizar las teorías por razones filosóficas, y partiendo de este supuesto, quisiera detectar los diversos sentidos que puede adquirir el término "axioma" en el caso de teorías empíricas; ellos corresponden, según creo, a otros tantos tipos de estructuras conceptuales implí. citas en las teorías. De todos modos, indirectamente mis indagaciones también pueden interesar a los detractores del método axiomático en filosofía de la ciencia: ya sea porque ellas les hagan ver que, después de todo, ciertamente pueden obtenerse algunas ideas valiosas sobre la estructura de la ciencia a través de la axiomatización, o bien porque al menos la discusión les sirva para afinar más en lo sucesivo sus argumentos anti-axiomáticos.

En lo que sigue voy a presuponer también que no sólo es conveniente desde un punto de vista ideal, sino además factible desde un punto de vista práctico, axiomatizar teorías empíricas. Puede que haya teorías que, por principio (y

1 Cf. C. U. Moulines, "Theoretical Terms and Bridge Principles: A Critique of Hempel's (Self-) Criticisms", Erkenntnis, vol. 22 (1985). 
no sólo por la pereza o por la confusión de los investigadores), no sean axiomatizables o que su axiomatización conduzca a resultados totalmente inadecuados. Es probable que, si tales teorías existen, debamos decir que su estado conceptual es "delicuescente" y que por tanto es muy difícil, cuando no imposible, someterlas a un análisis filosófico mínimamente articulado. Sea como sea, lo único que necesitamos admitir aquí es que el conjunto de las teorías de facto noaxiomatizables no abarca la totalidad de las teorías empíricas. Y creo que este supuesto no es una fantasía. El conjunto de las teorías físicas, económicas, fisiológicas, biológicas, lingüisticas y hasta literarias axiomatizadas hasta la fecha con un grado aceptable de rigor y adecuación es lo bastante numeroso como para que tengamos la seguridad de no estar hablando en el aire. Es posible dar ejemplos "cotidianos" de teorías empíricas axiomatizadas (ya sea por los propios científicos o por los filósofos). A este conjunto de teorías se refiere la indagación que sigue.

Una primera pregunta que podemos plantearnos al contemplar teorías empíricas axiomatizadas es: ¿tienen todos sus axiomas el mismo carácter? Aunque esta pregunta, así formulada, no es todavía muy clara, porque no sabemos exactamente lo que es el "carácter" de un axioma, intuitivamente parece manifiesto que la respuesta tendrá que ser negativa. Ahora bien, si los axiomas de una teoría empírica no tienen todos el mismo estatuto, entonces, ¿qué tipos de axiomas podemos distinguir y cuál es su función?

Consideremos un ejemplo particularmente sencillo, y no obstante real, de teoría empírica axiomatizada: la mecánica del choque. ${ }^{2}$ Ésta es una teoría que hoy día lleva una existencia más o menos "parasitaria" con respecto a la mecánica newtoniana de partículas. Sin embargo, inventada en

2 La axiomatización que sigue está basada, con algunas modificaciones, en la que W. Balzer y F. Mühlhölzer presentan en su articulo "Klassische Stossmechanik", Zeitschrift für allgemeine Wissenschaftstheorie, vol. XIII/1 (1982). 
lo esencial por Descartes y perfeccionada luego por Huygens, tuvo una existencia independiente y promisoria durante buena parte del siglo XVII, hasta que Newton la "absorbió" en sus Principia; por otra parte, todavía hoy día puede aplicarse a situaciones empíricas (por ejemplo, en física de partículas), en las que la aplicación de la mecánica newtoniana no viene al caso. Ambas razones, la histórica y la metodológica, nos justifican a tratarla como teoría con su propia "personalidad" (aunque, por supuesto, provista de nexos importantes con la mecánica newtoniana, en un sentido que luego explicaremos). El método axiomático del que hago uso aquí es el llamado de "axiomatización por definición de un predicado conjuntista". Este método es el más adecuado para discutir las cuestiones que aquí nos interesan. ${ }^{3} \mathrm{El}$ predicado conjuntista correspondiente a la mecánica (clásica) del choque es el siguiente:

$\mathrm{MCH}(\mathrm{x})$ si y sólo si: existen $\mathrm{P}, \mathrm{S}, \mathrm{T}, \mathrm{c}, \mathrm{v}, \mathrm{m}, \mathrm{t}, \mathrm{t}, \mathrm{t}$ tales que:

(0) $\mathrm{x}=\langle\mathrm{P}, \mathrm{S}, \mathrm{T}, / \mathrm{R}, \mathrm{c}, \mathrm{v}, \mathrm{m}>$

(1) $\mathrm{P}$ es un conjunto finito no-vacío

(2) $\mathrm{S}$ es un conjunto no-vacio

(3) $\mathrm{T}=\left\{\mathrm{t}_{1}, \mathrm{t}_{2}\right\}$

(4) c es una función biyectiva de $\mathrm{S}$ en $/ \mathrm{R}^{3}$

(5) v es una función de $\mathrm{P} \times \mathrm{T}$ en $\mathrm{S}$

(6) $m$ es una función de $P$ en $/ R$ tal que, para todo $p^{\varepsilon P}$, $m(p)>0$

(7) $\sum_{p \varepsilon P} m(p) \cdot c^{\circ} v\left(p, t_{1}\right)=\sum_{p \varepsilon P} m(p) \cdot c^{\circ} v\left(p, t_{2}\right)$

(donde '/R' designa el conjunto de números reales y ${ }^{\circ}$, la operación de composición de funciones).

3 Una exposición detallada del método en cuestión se hallará en el capítulo final de P. Suppes, Introducción a la lógica, CECSA, México, 1964. Sobre las ventajas de este método con respecto al de axiomatización formal a lo HilbertCarnap, puede consultarse W. Stegmüller, La concepción estructuralista de las teorias, Alianza Universidad, Madrid, 1981. 
Las estructuras del tipo indicado en la condición (0) que satisfagan el predicado MCH son los modelos de la mecánica del choque. En primera instancia podemos decir que las condiciones (1)-(7) son los axiomas de la mecánica del choque. (7) representa su "ley fundamental", a saber, la ley de la conservación del momento. Aparte de $/ R$, que es una entidad matemática con interpretación fija para todos los modelos, los demás miembros de cada estructura $\mathrm{x}(\mathrm{P}$, $\mathrm{S}, \mathrm{T}, \mathrm{c}, \mathrm{v}, \mathrm{m}$ ) son los llamados "conceptos primitivos" o "básicos" de la teoría; en realidad, no son conceptos, en el sentido usual, sino esquemas de conceptos: puesto que se introducen como variables cuantificadas en la definición general de $\mathrm{MCH}$, pueden recibir las interpretaciones más diversas según el modelo. Así, por ejemplo, podríamos sustituir la variable $P$ por $\{1,2\}, S$ por $/ R^{3}, T$ por $\{1,2\}$, c por la función idéntica, $\mathrm{v}$ por $\{\langle 1,1,1\rangle,\langle 2,1,2\rangle,\langle 1,2,1\rangle$, $<2,2,2\rangle\}$ y m por $\{\langle 1,1\rangle,\langle 2,2\rangle\}$. Es fácil constatar que, bajo esta interpretación, quedan satisfechas las condi. ciones de definición de MCH, inclusive (7). Pero está claro que nadie dirá que el modelo propuesto representa un choque físico, para empezar porque $\mathrm{P}$ debe entenderse como un conjunto de objetos físicos ("partículas") y no como un conjunto de números. Habrá pues una gran cantidad (de hecho: un número infinito) de modelos de $\mathrm{MCH}$ que no nos interesan en absoluto, no forman realmente parte de la teoría. Saber esto es también saber algo acerca de la identidad de la mecánica del choque, algo que no está incluido en las condiciones (1)-(7). Este algo adicional viene fijado de manera informal por enunciados como los siguientes:

(a) Los elementos de $\mathrm{P}$ son partículas.

(b) $\mathrm{S}$ es una región del espacio físico (clásico).

(c) T está constituido por dos instantes.

(d) c es una coordinatización del espacio físico. 
(e) v es la velocidad de cada partícula en cada instante.

(f) m es la masa de cada partícula.

Estos enunciados no se deducen de lo que sabíamos de MCH hasta aquí. Es más, en ellos aparecen términos, como 'partícula', 'espacio físico', etcétera, que ni pertenecen al vocabulario de la matemática pura ni forman parte de los modelos de $\mathrm{MCH}$. Por su carácter está claro que tampoco tendría sentido añadirlos simplemente a la lista de condiciones de definición de $\mathrm{MCH}$, pues con ello sólo obtendríamos aburridas tautologías. Llamemos a (a)-(f) 'axiomas de interpretación' de MCH. Estos principios también forman parte de la identidad de la mecánica del choque, pero su estatuto es distinto de (1)-(7). Su función consiste en subsumir cada uno de los conceptos primitivos de $\mathrm{MCH}$ bajo cierto género de cosas. Por ejemplo, se dice que los elementos de P pertenecen al género de cosas llamadas 'partículas' $\mathrm{y}$ que $\mathrm{v}$ pertenece al género de funciones llamadas 'velocidades'. ¿Dónde aparecen determinados tales géneros? No dentro de la MCH misma, por supuesto. Parece que hay filósofos que sostienen que estos géneros vienen determinados sin necesidad de ninguna teoría, por nuestro conocimiento directo de la realidad. Por una especie de intuición cuasimística sabríamos directamente, sin mayor aditamento, a qué se refiere 'partícula' y a qué se refiere 'velocidad'. Los axiomas de interpretación serían así una especie de dedos semánticos (sustitutos de los dedos de carne y hueso) que llevarían directamente de las palabras a "la Realidad". Esta idea proviene de una concepción típicamente metafísica en el mal sentido, es decir, inextricablemente confusa. La confusión proviene de una mezcla indigerible de puntos de vista psicológicos, epistemológicos y lógicos en el análisis de las teorías. Sean cuales sean las imágenes que nos provoquen en el cerebro los términos 'partícula' o 'velocidad' (por ejemplo, la imagen de una bolita muy pequeña o de algo 
que se mueve), ellas no vienen al caso cuando se trata de analizar el papel que juegan estos conceptos en la estructura y el funcionamiento de una teoría como la mecánica del choque. La misión de los axiomas de interpretación, en todo caso, no consiste en proporcionarnos un "contacto estrecho de tercera especie" con la apenas vislumbrada Realidad Pura, sino en indicarnos las conexiones de la teoría en cuestión con otras teorias, que le proporcionan por así decir su susiento metodológico, que son sus presupuestos. Estas teorías, en la mayoría de los casos, son perfectamente identificables e incluso están ya axiomatizadas o cuasi-axiomatizadas. Por ejemplo, en nuestro caso, el axioma de interpretación (b) nos indica, a través del concepto de espacio físico, que la mecánica del choque necesita vitalmente de un vínculo con la geometría física euclídea (teoría ya axiomatizada); (c) nos indica que necesita asimismo de la cronometría (otra teoría identificable); (d) y (e) nos remiten a la cinemática clásica (galileana); (f) apunta hacia la mecánica newtoniana de partículas y hacia la mecánica del sólido rígido (dos teorías archi-axiomatizadas). El lector habrá notado que, astutamente, he dejado para el final el comentario sobre el axioma de interpretación (a). Es cierto que no disponemos (por lo que sé) de una teoría bien definida que determine el concepto de partícula. Pero ello no es, a mi entender, debido a que dispongamos de una intuición directa de lo que es una partícula, sino a que la teoría correspondiente a esa noción es probablemente de estructura tan elemental que nadie se preocupa por explicitarla. De todos modos, en filosofía de la ciencia, como en todas partes, a veces se da uno grandes sorpresas y quizás valdría la pena reconstruir detalladamente la teoría que está detrás del concepto de partícula (de la física clásica, claro). Posiblemente baste con algún tipo de mereología, a la que eventualmente se pueda aplicar el método de la abstracción extensiva que introdujo 
Whitehead en la construcción de volúmenes puntuales. ${ }^{4}$ Sea como sea, espero que esta discusión haya mostrado suficientemente que la función de los axiomas de interpretación es establecer una especie de vínculos interteóricos, por así decir puntuales y asimétricos, que transmiten la sangre de una teoría a la otra. Admito que, como caracterización general, todo ello aún no es todo lo claro que debería ser. Ciertamente, se puede y se debe explicitar la forma lógica de tales principios. Sobre ello volveré más adelante. Todavía necesitamos antes un poco de aparato para precisar las ideas.

Def. O: Llamaremos 'vocabulario formal' de una teoría em. pírica al conjunto de símbolos que aparecen en ella provenientes de la lógica de predicados de primer orden con identidad, más signos auxiliares, más ' $\epsilon$ ', más todos los símbolos que puedan definirse mediante los anteriores. Llamaremos 'vocabulario básico' al conjunto de los términos que denotan las entidades que constituyen los modelos de una teoría ( = los miembros de los tuplos correspondientes en la axiomatización por predicado conjuntista).

Entre los conceptos conjuntistas "puros", es decir, aquellos que son definibles mediante uso exclusivo de $\epsilon$ y de la lógica de predicados, están algunos que necesitamos para nuestro análisis y cuya expresión simbólica conviene, por tanto, tener presente. Son los siguientes:

—_ ' $\subseteq$ ' Para la relación de inclusión;

— ' $\subset$ ' para la relación de inclusión propia;

_ $\cup$ ' para la gran unión de conjuntos;

__ 'P' para el conjunto-potencia;

4 Las posibles aplicaciones empíricas del método de la abstracción exten. siva se discuten en C. U. Moulines, La estructura del mundo sensible, Ariel, Barcelona, 1973, pp. 110 y ss. 
_. ' $X$ ' para el producto cartesiano;

'<...>' para los elementos de un producto cartesiano;

'Func' para el concepto general de función;

' $\mathrm{f}: \mathrm{C} \longrightarrow \mathrm{D}$ ' indica que el dominio de la función $\mathrm{f}$

es $\mathrm{C}$ y su contradominio un subconjunto de $\mathrm{D}$;

$\longrightarrow{ }^{\prime} \mathrm{C} \longleftrightarrow D$ ' indica que el conjunto $\mathrm{C}$ es biyectable con el conjunto $\mathrm{D}$;

' $\varnothing$ ' para el conjunto vacío;

'A - B' para indicar la diferencia entre los conjuntos A y B.

Necesitamos, además, otro concepto auxiliar conjuntista, que no es tan usual como los anteriores:

Def. 1: Si $\mathrm{x}=\left\langle\mathrm{a}, \ldots, \mathrm{a}_{\mathrm{n}}\right\rangle$, entonces, para $\mathrm{i} \leqslant \mathrm{n}, \pi_{\mathrm{ix}}$ $=$ ai. $\pi_{i}$ es pues una función de tuplos en miembros de tuplos. La denominamos "proyección-i".

Def. 2: Sea $A$ una fórmula cualquiera del lenguaje de la teoría de conjuntos. $\mathrm{Y}$ sea ' $t$ ' un término de ese lenguaje que denota un conjunto cualquiera. Entonces ' $A[t]$ ' expresa que ' $t$ ' ocurre esencialmente en $A$ y ' $A(t)$ ' ha de expresar que, aparte del vocabulario formal y (eventualmente) de las variables ligadas, ' $t$ ' es el único término conjuntista que ocurre esencialmente en dicha fórmula:

$A(t)$ si y sólo si $A[t]$ y, para todo $t^{\prime}$ del vocabulario básico, si $A\left[t^{\prime}\right]$, entonces $t=t^{\prime}$.

Nos interesa ahora definir lo que es la tipificación de una entidad conjuntista en función de otras entidades conjunlistas dadas. Intuitivamente hablando, las tipificaciones indican el nivel conjuntista en el que se ha construido un conjunto o una relación determinados; indican, por así decir, su nivel ontológico dentro de la reconstrucción conjuntista de la teoría. Ello resulta imprescindible para un análisis 
conceptual de las teorías (para saber de qué tipo de conceptos estamos hablando) y también para el análisis de las relaciones globales entre teorías, en particular lo que he llamado en otra parte 'reducción ontológica'. 'Las tipificaciones constituyen lo primero que hay que saber acerca de una teoría. Primero hay que definir lo que es un tipo o, más exactamente, lo que es un tipo-k. La definición es recursiva.

Def. 3: Para cada $\mathrm{k} \in / \mathrm{N}$ :

(1) Si $\mathrm{i} \leqslant \mathrm{k}$, entonces $\pi_{1}$ es un tipo-k

(2) si $\tau$ es un tipo-k, entonces $P(\tau)$ es un tipo-k

(3) si $\tau$ y $\sigma$ son tipos-k, entonces $\tau \times \sigma$ es un tipo-k Def. 4: Dadas las entidades conjuntistas $\mathrm{C}, \mathrm{D}_{1}, \ldots, \mathrm{D}_{\mathrm{k}}$, diremos que la fórmula $A$ es una tipificación de C con respecto a $D_{1}, \ldots, D_{k}$ si y sólo si existe un tipo-k $\tau$ tal que $A$ es de la forma

$$
\text { ' } \mathrm{C} \in \tau\left(\mathrm{D}_{1}, \ldots, \mathrm{D}_{\mathrm{k}}\right) \text { ' }
$$

La tipificación de una entidad conjuntista (por ejemplo una relación) en función de otras entidades consiste en una indicación de cómo está construida dicha entidad paso a paso a partir de las entidades de base asumidas. Para ello se necesita solamente de las operaciones conjuntistas de la proyección, el conjunto-potencia y el producto cartesiano. Ellas nos bastan para fijar el nivel ontológico de una entidad cualquiera en una teoría dada. Veamos un ejemplo particularmente sencillo de tipificación. Supongamos que queremos introducir cierta entidad $\mathrm{E}$ como relación diádica entre dos conjuntos de base, previamente dados, $\mathrm{D}_{1}$ y $\mathrm{D}_{2}$. Para ello se escribe usualmente:

5 Cf. C. U. Moulines, "Ontological Reduction in Natural Science". En: Reduction in Science (comp. por W. Balzer, D. Pearce, H. J. Schmidt), Reidel, 1984. 
Pues bien, esta fórmula contiene implícitamente tres pasos "tipificadores":

19) Tomamos $\left\langle D_{1}, D_{2}\right\rangle$ y aplicamos $\pi_{1}, \pi_{2}$ : $\pi_{1}<\mathrm{D}_{1}, \mathrm{D}_{2}>=\mathrm{D}_{1}, \pi_{2}<\mathrm{D}_{1}, \mathrm{D}_{2}>=\mathrm{D}_{2}$

$2^{\text {9) }}$ Construimos $\mathrm{D}_{1} \times \mathrm{D}_{2}$, o sea, $\left.\left.\pi_{1}<\mathrm{D}_{1}, \mathrm{D}_{2}\right\rangle \times \pi_{2}<\mathrm{D}_{1}, \mathrm{D}_{2}\right\rangle$ $3^{\circ}$ ) Construimos $\mathcal{P}\left(\mathrm{D}_{1} \times \mathrm{D}_{2}\right)$, o sea, $\mathcal{P}\left(\pi_{1}<\mathrm{D}_{1}, \mathrm{D}_{2}>\times \pi_{2}\right.$ $\left.<\mathrm{D}_{1}, \mathrm{D}_{2}\right\rangle$ )

$\mathrm{Y}$ tipificamos finalmente $\mathrm{E}$ como

$$
(* *) \quad E \in P\left(\pi_{1}<D_{1}, D_{2}>\times \pi_{2}<D_{1}, D_{2}>\right),
$$

que equivale a la humilde fórmula $(*)$.

Por supuesto que en la práctica preferiremos escribir $(*)$ a $\left(^{* *}\right)$. Pero la ventaja de $(* *)$ sobre $(*)$ es de orden conceptual: nos indica claramente cómo está construida la relación a partir de los conjuntos de base. Nos dice: si se quiere obtener la relación en cuestión, tómese primero el conjunto $\mathrm{D}_{1}$, luego el $\mathrm{D}_{2}$, constrúyase su producto cartesiano y finalmente considérese el conjunto-potencia del mismo; la relación apetecida será un elemento de dicho constructo. Es este carácter "constructivo" de las tipificaciones lo que interesa en una reconstrucción axiomática de las teorías y también, como he indicado más arriba, en el análisis de relaciones interteóricas como la reducción.

Las tipificaciones constituyen la porción más básica de la axiomatización de una teoría; son, por así decir, su aspecto absolutamente a priori. Aunque se trate de una teoría empírica, las tipificaciones no tienen el menor carácter de enunciados empíricos: su misión consiste exclusivamente en explicitar el tipo de aparato conceptual que queremos usar. Carece por completo de sentido tratar de confirmar o refutar 
una tipificación. Lo único que podemos tratar de averiguar es si una tipificación propuesta es realmente adecuada para el concepto que queremos introducir; pero ésta es una cuestión de puro análisis conceptual. Además, las tipificaciones tienen el efecto de deslindar claramente lo que son los conjuntos de base de la teoría (= la "ontología básica") por un lado de las relaciones y funciones construidas sobre ellos por otro. En el caso de las teorías empíricas matematizadas necesitaremos generalmente de dos clases de conjuntos de base: aquellos cuyos elementos son individuos empíricos y que vienen interpretados por ciertos axiomas de interpretación en el sentido explicado más arriba y aquellos que son entidades matemáticas de interpretación fija (por ejemplo los números naturales o los números reales). Para fijar ideas, llamaremos al primer tipo de conjuntos de base "dominios (de individuos)" y a los segundos "conjuntos auxiliares". Habitualmente, las tipificaciones se construirán sobre una mezcla de algunos dominios con algunos conjuntos auxiliares; pero en cualquier caso, una teoría empírica se caracteriza por el hecho de que en toda tipificación deberá aparecer al menos un dominio de individuos.

Veamos cómo funciona esta idea en el ejemplo de MCH. Según lo dicho, necesitamos tipificaciones para las entidades conjuntistas de carácter relacional o funcional. En nuestro ejemplo, ellas son: c, v, m. Los dominios son P, S, T y el único conjunto auxiliar es $/ \mathrm{R}$. Las tipificaciones correspondientes están "escondidas" en las condiciones (4)-(6) :

$$
\begin{aligned}
\text { (T-I) } & \mathrm{c} \in \mathrm{P}\left(\pi_{1}<\mathrm{S}, / \mathrm{R}>\times \pi_{2}<\mathrm{S}, / \mathrm{R}>\times \pi_{2}<\mathrm{S}, / \mathrm{R}>\times \pi_{2}\right. \\
& <\mathrm{S}, / \mathrm{R}>) \\
\text { (T-II) } & \mathrm{v} \in \mathrm{P}\left(\pi_{1}<\mathrm{P}, \mathrm{T}, \mathrm{S}>\times \pi_{2}<\mathrm{P}, \mathrm{T}, \mathrm{S}>\times \pi_{3}<\mathrm{P}, \mathrm{T}, \mathrm{S}>\right) \\
\text { (T-III) } & \mathrm{m} \in \mathrm{P}\left(\pi_{1}<\mathrm{P}, / \mathrm{R}>\times \pi_{2}<\mathrm{P}, / \mathrm{R}>\right)
\end{aligned}
$$

Por mínimo y trivial que sea el contenido informativo de una teoría empírica, ella nos debe hablar por lo menos de 
alguna relación entre un par de conjuntos de base. Si sólo tuviéramos conjuntos de base sin ninguna relación, no sabriamos qué hacer con ellos; en el sentido usual de "estructura" podríamos decir que la teoría carece de estructura. Y una teoría sin estructura es como un disco sin surco: no dice absolutamente nada. Por ello, toda teoría empírica que se precie mínimamente debe introducir por lo menos una relación entre sus conceptos primitivos. Y esta relación deberá estar tipificada en función de los conjuntos de base, de lo contrario no podríamos hacer nada con ella (dentro de esa teoría). De ahí proviene mi

\section{Primera tesis axiomatológica:}

Entre los axiomas de una teoría empírica figura al menos una tipificación.

Ahora bien, está claro que las tipificaciones $(T$-I $) \cdot(T$-III $)$ no agotan el contenido de los enunciados primigenios (4) -(6) correspondientes que las engloban. El resto de contenido informativo que poseen (4)-(6) no puede expresarse como tipificación, puesto que el carácter unívoco por la derecha de una relación o su positividad no pueden expresarse como construcciones a partir de los conjuntos de base mediante uso exclusivo de los "escalones" $\pi_{\mathrm{i}}, \mathcal{P}, \mathrm{x}$. Esto es aún más obvio, si cabe, para la totalidad de los enunciados (1) -(3) y (7). ¿Cuál es el estatuto lógico-metodológico de estos axiomas?

Dejemos de momento (7) como caso aparte. Para empezar, el rasgo notorio de (1)-(3) es que, en cada una de las fórmulas, aparte del vocabulario formal hay un solo término del vocabulario básico que ocurre en ellas esencialmente: el término correspondiente a un dominio en cada caso. ${ }^{6}$ Algo análogo puede decirse de las condiciones (4)-(6), con o sin

${ }^{6}$ Nótese que para expresar la finitud de $\mathrm{P}$ no es necesario recurrir a los números naturales: basta con requerir que ningún subconjunto propio de $\mathrm{P}$ sea biyectable con $P$. Nótese también que $t_{1} y t_{2}$ en $(3)$ son variables ligadas. 
tipificaciones: cada una de ellas se refiere unívocamente a una relación, sólo que aquí, además de la relación en cuestión intervienen los conjuntos de base que tipifican la relación. Las condiciones (1)-(6), pues, en la medida en que no son tipificaciones, son estipulaciones que caracterizan las propiedades matemáticas esenciales de cada uno de los términos. Por ejemplo, dicen que algo no es vacío, o que es unívoco por la derecha, o que es siempre positivo. No son tan claramente apriorísticas (al menos en algunos casos) como las tipificaciones, que simplemente fijan el nivel ontológico de cada uno de los componentes de nuestro aparato conceptual. De algún modo reflejan ciertos hechos que podríamos imaginar que fueran de otra manera, incluso asumiendo el mismo tipo de aparato conceptual. Por ejemplo, podríamos imaginar que, en algún caso, para describir un choque necesitáramos asumir la existencia de un número infinito de partículas, o que, en otro caso, fuera conveniente asignar a algunas partículas masas negativas. Las caracterizaciones tienen, pues, aunque sea en grado mínimo, un cierto contenido factual. Sin embargo, no se trata de enunciados directamente empíricos como pueda ser la afirmación de la negrura de los cuervos. Y, sobre todo, su contenido empírico es muy pobre porque siempre se refieren sólo a un concepto. Llamemos a estos eslabones perdidos entre lo a priori y lo $a$ posteriori de una teoría 'caracterizaciones matemáticas de los conceptos básicos', o para abreviar, 'caracterizaciones' a secas. Las podemos definir así:

Def. 5: Sean $t_{1}, \ldots, t_{\mathrm{h}} \mathrm{h}$ términos básicos de una teoría $\mathrm{T}$. Diremos que $A\left(t_{1}, \ldots, t_{\mathrm{h}}\right)$ es una caracterización (en T) si y sólo si $A\left(t_{1}, \ldots, t_{\mathrm{n}}\right)$ no es una tipificación y (a) $\mathrm{h}=1$, o bien

(b) existe un y sólo un $t_{\mathrm{j}} \in\left\{t_{1}, \ldots, t_{\mathrm{h}}\right\}$ tal que $t_{\mathrm{j}}$ está tipificado por $\left\{t_{1}, \ldots, t_{\mathrm{n}}\right\}-\left\{\mathrm{t}_{\mathrm{j}}\right\}$ 
Dado que en toda teoría empírica, por elemental que sea, debe haber por lo menos un conjunto de base que sea un dominio, se infiere que en la axiomatización de toda teoría empírica deberá estar incluida al menos una caracterización.

Segunda tesis axiomatológica:

Entre los axiomas de una teoría empírica figura al menos una caracterización.

En las teorías empíricas con un mínimo de complejidad matemática (de hecho: cualquier teoría empírica que pretenda servir para algo) no sólo será preciso caracterizar los dominios, sino también las relaciones tipificadas sobre los dominios, porque las tipificaciones de estas últimas no serán un medio suficiente para determinar su estructura conceptual. La parte de las condiciones (4)-(6) de MCH que no aparece expresada en ( $T$-1)-(T-III) es un ejemplo de tales caracterizaciones relacionales.

¿Qué pasa con (7)? La ley de la conservación del momento expresada en (7) no es evidentemente una tipificación, ni tampoco una caracterización: es fácil comprobar que ese enunciado no satisface la Def. 5, pues en él ocurren esencialmente los tres términos funcionales de la teoría, aparte del conjunto de base $\mathrm{P}$. La ley de la conservación del momento es justamente eso: una ley. $O$, para ser más precisos, puesto que quizá a las caracterizaciones, por lo menos a algunas de ellas, también se las podría calificar de leyes, diremos que (7) pertenece a una categoría particular de leyes: las leyes sinópticas. En sentido más o menos vago, las leyes sinópticas son los axiomas "más importantes" de una teoría, los que contienen la "información esencial". Se caracterizan por ser enunciados sumamente sintéticos que ponen en conexión mutua todos los términos relacionales del vocabulario básico de la teoría y (explícita o implícitamen- 
te) los conjuntos de base. Sin leyes sinópticas, una teoría consistiría solamente en las piezas de un rompecabezas, piezas más o menos curiosas y complicadas, pero piezas aisladas al fin; es tan sólo la ley sinóptica la que logra poner orden en ese conjunto de elementos dispares combinándolos en un cuadro armonioso. Por ello, también las leyes sinópticas son imprescindibles a una teoría que se precie.

Def. 6: Una fórmula $A$ es una ley sinóptica de la teoría $T$ si y sólo si, para todo término $t$ del vocabulario básico de $T$ tipificado en $T$, se cumple: $A[t]$.

Con base en esta definición asentaré mi

Tercera tesis axiomatológica:

Entre los axiomas de una teoría empírica figura al menos una ley sinóptica.

Disponemos ya de bastantes ejemplos de leyes sinópticas en las teorías axiomatizadas hasta la fecha por el método del predicado conjuntista; además de la ley de la conservación del momento, otros ejemplos famosos en física son: el segundo principio de Newton, la ecuación fundamental de Gibbs, la ley de Euler en hidrodinámica, "Ias ecuaciones" de Lagrange y la ley de Ohm. ${ }^{7}$

Aplicando la tricotomía tipificación-caracterización-ley sinóptica a nuestro ejemplo de la mecánica del choque, podemos volver a definir el predicado conjuntista utilizando esa distinción tripartita. El resultado es una axiomatización de

7 Para constatar el carácter sinóptico de las dos primeras leyes mencionadas, cf. C. U. Moulines, Exploraciones metacientificas, Alianza Universidad, Madrid, 1982; para la tercera, cf. J. Flematti, "A Logical Reconstruction of the Hydrodynamics of Ideal Fluids", UNAM, México, 1984; para la cuarta, cf. J. D. Sneed, The Logical Structure of Mathematical Physics, Reidel, 1971/ 1978; para la quinta, cf. M. Heidelberger, Der Wandel der Elektrizitätslehre zu Ohms Zeit, Munich, 1979. 
apariencia más pedante, pero que revela exactamente la naturaleza de los axiomas requeridos:

$\mathrm{MCH}(\mathrm{x})$ si y sólo si existen $\mathrm{P}, \mathrm{S}, \mathrm{T}, \mathrm{c}, \mathrm{v}, \mathrm{m}, \mathrm{t}_{1}$, $\mathrm{t}_{2}$ tales que:

(O) $\mathrm{x}=\langle\mathrm{P}, \mathrm{S}, \mathrm{T}, / \mathrm{R}, \mathrm{c}, \mathrm{v}, \mathrm{m}\rangle$

(T-I) $\mathrm{c} \in \mathcal{P}\left(\pi_{1}<\mathrm{S}, / \mathrm{R}>\times \pi_{2}<\mathrm{S}, / \mathrm{R}\right) \times \pi_{2}<\mathrm{S}, / \mathrm{R}>$ $\left.\times \pi_{2}<\mathrm{S}, / \mathrm{R}>\right)$

(T-II) $v \in \mathcal{P}\left(\pi_{1}<\mathrm{P}, \mathrm{T}, \mathrm{S}>\times \pi_{2}<\mathrm{P}, \mathrm{T}, \mathrm{S}>\times \pi_{3}<\mathrm{P}, \mathrm{T}, \mathrm{S}>\right)$

(T-III) $\mathrm{m} \in \mathcal{P}\left(\pi_{1}<\mathrm{P}, / \mathrm{R}>\times \pi_{2}<\mathrm{P}, / \mathrm{R}>\right)$

$(C$-I $) \mathrm{P} \neq \varnothing \& \sim \exists \mathrm{P}^{\prime}\left(\mathrm{P}^{\prime} \subset \mathrm{P} \& / / \mathrm{P} / /=/ / \mathrm{P}^{\prime} / /\right)$

(C-II) $\mathrm{S} \neq \varnothing$

(C-III) $\mathrm{T}=\left\{\mathrm{t}_{1}, \mathrm{t}_{2}\right\}$

(C-IV) Func(c)

(C-V) Func(v)

$(C$-VI) Func $(\mathrm{m}) \& \forall p \in \mathrm{P}(\mathrm{m}(\mathrm{p})>0)$ $(S-I) \sum_{p \in P} \mathrm{~m}(\mathrm{p}) \cdot \mathrm{c}^{\mathrm{o} v}\left(\mathrm{p}, \mathrm{t}_{1}\right)=\sum_{\mathrm{p} \in \mathrm{P}} \mathrm{m}(\mathrm{p}) \cdot \mathrm{c}^{\mathrm{0} v}\left(\mathrm{p}, \mathrm{t}_{2}\right)$

En una teoría tan elemental como la mecánica del choque hemos destilado tres tipos de axiomas (tipificaciones, caracterizaciones y leyes sinópticas) y hemos determinado su forma lógica. Pero nuestra tarea de disección axiomática aún no ha concluido. Recordemos que habíamos prometido decir algo más sustancioso sobre los llamados "axiomas de interpretación". Recordemos también que habíamos insinuado que se trataba (implícitamente) de relaciones interteóricas de "sentido único" (como en las reglas de tránsito) entre $\mathrm{MCH}$ y otras teorías, por así decir, más básicas. ¿Cómo podemos precisar esta idea? A alguien se le podría ocurrir en este punto tratar de ahorrarse trabajo arguyendo que si los axiomas de interpretación son relaciones interteóricas, entonces su dilucidación lógica no corresponde al "morfólogo" que analiza una teoría, sino acaso el "ecólogo" que se ocupa de 
"nichos" de teorías en mutua relación. Esta objeción sería apropiada desde el punto de vista de las teorías presupuestas por MCH en su interpretación. Sin duda, la geometría física o la cinemática pueden vivir perfectamente ignorando la existencia de la mecánica del choque; en un armonioso mundo sin choques podrían seguir siendo válidas. Pero la inversa no es cierta. La mecánica del choque necesita vitalmente de los conceptos que le proporcionan la geometría física, la cinemática y (hoy día) la mecánica newtoniana. ${ }^{8}$ Su identidad misma depende de estas teorías, por lo que no podemos hacernos la vida fácil y descuidar los axiomas de interpretación. Introduzcamos un par más de nociones técnicas que nos aliviarán la tarea.

La clase M[MCH] de modelos de la mecánica del choque es la clase de estructuras conjuntistas que satisfacen el predicado $\mathrm{MCH}$, es decir, que cumplen las tipificaciones ( $T$-I) - ( $T$-III), las caracterizaciones $(C$-I $)-(C$-VI) y la ley sinóptica $(S-I)$. Si suprimimos de nuestra consideración esta última, obtendremos estructuras compuestas por una serie de piezas conceptuales aisladas que no sabemos si, y cómo, armonizan entre sí. La clase de estructuras con esta propiedad es evidentemente "mayor" que la clase de los modelos. Para seguir la tradición, a esa clase de estructuras la llamaremos 'clase de los modelos potenciales de MCH', abreviadamente: $\mathrm{MP}[\mathrm{MCH}]$. No hace falta romperse la cabeza para comprender que se cumple:

$$
\mathrm{M}[\mathrm{MCH}] \subseteq \mathrm{MP}_{\mathrm{P}}[\mathrm{MCH}]
$$

8 Esto último no era cierto para la $\mathrm{MCH}$ en su estatuto anterior a 1685. En aquella época, la masa (introducida por Huygens en la $\mathrm{MCH}$ ) no necesitaba ser interpretada en función de la mecánica newtoniana sencillamente porque esta última aún no existía. En aquella época, la masa de $\mathrm{MCH}$ era un concepto $\mathrm{MCH}$-teórico en el sentido de Sneed y por tanto no estaba interpretada en cada modelo, sino que era una variable introducida por un enunciado de Ramsey generalizado. (Sobre esta última noción, cf. W. Stegmüller, op. cit.) 
Pues bien, los axiomas de interpretación prescinden de si los conceptos de velocidad, masa, etcétera, combinados adecuadamente cumplen la conservación del momento; esto no les interesa en absoluto. Por consiguiente, en la teoría se insertarán no al nivel de $\mathrm{M}[\mathrm{MCH}]$, sino al nivel de $\mathrm{M}_{\mathrm{P}}[\mathrm{MCH}]$ (= afectan al "aparato conceptual en sí" antes que a las "leyes"). Pero, en cambio, al decirnos que v debe ser "realmente" la velocidad de la cinemática (es decir, una teoría que combina posición y tiempo según ciertas leyes) y que m debe ser "realmente" la masa de la mecánica newtoniana (es decir, de una teoría que combina fuerza, masa, posición y tiempo según otras leyes determinadas), están presuponiendo la validez total de esas teorías, es decir, incluidas sus leyes. Con todo este párrafo farragoso no quiero decir otra cosa sino que, en general, los axiomas de interpretación son la expresión de una relación entre los modelos potenciales de una teoría y los modelos (actuales) de otra.

Def. 7: Una fórmula $A\left(t, t^{\prime}{ }_{1}, \ldots, t^{\prime}{ }_{n}\right)$ es un axioma de interpretación de la teoría $T$ si y sólo si existen $\mathrm{i}, \mathrm{j} 1, \ldots, \mathrm{j}_{\mathrm{n}}, \mathrm{x}, \mathrm{x}^{\prime}, T^{\prime}$ tales que:
a) $t$ es un término del vocabulario básico de $T$
b) $t^{\prime}{ }_{1}, \ldots t^{\prime}{ }_{n}$ son términos del vocabulario básico de $T^{\prime}$
c) $\mathrm{i}, \mathrm{j}_{1}, \ldots, \mathrm{j}_{\mathrm{n}} \in / \mathrm{N}$
d) $\mathrm{x} \in \mathrm{Mr}[T]$
e) $x^{\prime} \in M\left[T^{\prime}\right]$
f) $\pi_{\mathrm{i}} \mathrm{x}=t$
g) $\pi_{\mathrm{j}_{1}} \mathrm{x}^{\prime \prime}=t_{1}^{\prime}, \ldots, \pi_{\mathrm{j}_{\mathrm{n}}} \mathrm{x}^{\prime}=t_{\mathrm{n}}^{\prime}$

De una manera mucho más sintética, aunque menos informativa, podríamos decir que un axioma de interpretación de $T$ en función de $T$ ' expresa una relación $V$ (un "vínculo") del tipo: 
Def. $7^{\prime}: V \subseteq \mathrm{M}[T] \times \mathrm{M}\left[T^{\prime}\right]$

Dado que, sin interpretación de sus conceptos básicos, no sabemos de qué nos habla una teoría empírica, inferimos nuestra

\section{Cuarta tesis axiomatológica:}

Entre los axiomas de una teoría empírica figura al menos uno que expresa un vínculo entre los modelos potenciales de esa teoría y los modelos actuales de otra.

Retornemos a nuestro querido ejemplo. Utilicemos las siguientes abreviaciones: '?' significa una teoría que aún no conocemos: 'GE' representa la geometría (física) euclídea; 'CR', la cronometría; 'KIN', la cinemática (galileana); 'MNP', la mecánica newtoniana de partículas; 'MSR', la mecánica del sólido rígido. Entonces, los axiomas de interpretación de la mecánica del choque que habíamos identificado unas páginas atrás, pueden sintetizarse en los postulados: ${ }^{9}$

$$
\begin{aligned}
(I-\mathrm{I}) & \exists V_{1}\left(V_{1} \subseteq \mathrm{M}_{\mathrm{P}}[\mathrm{MCH}] \times \mathrm{M}[?]\right) \\
(I-\mathrm{II}) & \exists V_{2}\left(V_{2} \subseteq \mathrm{M}_{\mathrm{P}}[\mathrm{MCH}] \times \mathrm{M}[\mathrm{GE}]\right) \\
(I-\mathrm{III}) & \exists V_{3}\left(V_{3} \subseteq \mathrm{M}_{\mathrm{P}}[\mathrm{MCH}] \times \mathrm{M}[\mathrm{CR}]\right) \\
(I-\mathrm{IV}) & \exists V_{4}\left(V_{4} \subseteq \mathrm{M}_{\mathrm{P}}[\mathrm{MCH}] \times \mathrm{M}[\mathrm{KIN}]\right) \\
(I-\mathrm{V}) & \exists V_{5}, V_{6}, \mathrm{M}_{1}, \mathrm{M}_{2}\left(\mathrm{M}_{1} \subseteq \mathrm{M}_{\mathrm{P}}[\mathrm{MCH}]\right. \\
& \& \mathrm{M}_{2} \subseteq \mathrm{M}_{\mathrm{P}}[\mathrm{MCH}] \& V_{5} \subseteq \mathrm{M}_{1} \times \mathrm{M}[\mathrm{MNP}] \\
& \left.\& V_{6} \subseteq \mathrm{M}_{2} \times \mathrm{M}[\mathrm{MSR}]\right)
\end{aligned}
$$

El último axioma de interpretación pretende expresar que la masa que se utiliza en MCH en algunos casos (= modelos)

9 La explicación completa de estos axiomas con mención de los términos vinculados en cada caso consistiría en una mera formalización, siguiendo la pauta de la Def. 7, de los enunciados (a)-(f) formulados páginas atrás. Me ahorro aquí tal repetición, pareciéndome más interesante presentar su expresión "abstracta" modelo-teórica. 
se determina presuponiendo la mecánica newtoniana de partículas $\mathrm{y}$, en otros, presuponiendo la mecánica del sólido rígido. Si hubiera otros modos "clásicos" de determinar la masa que interviene en un choque bastaría añadir en $(I-\mathrm{V})$ los vínculos $V_{\imath}$, etcétera, correspondientes. Por supuesto que a veces, la masa de una partícula que se presupone en el estudio de un choque no proviene ni de la mecánica newtoniana de partículas, ni de la del sólido rígido, sino de la misma mecánica del choque, aunque de otro modelo distinto de aquel que se está considerando. Examinemos esta situación más detalladamente.

Supongamos que hacemos chocar la partícula $p_{1}$ a velocidad inicial $c^{0} v\left(p_{1}, t_{1}\right)=v_{1}$ con la partícula $p_{2}$, de la que hemos constatado la velocidad inicial $c^{0} v\left(p_{2}, t_{1}\right)=v_{2}$. Después del choque "observamos" (es decir, determinamos por medios cinemáticos) las velocidades respectivas $c^{0} v\left(p_{1}, t_{2}\right)$ $=\mathrm{v}^{\prime}{ }_{1}$ y c $^{0} \mathrm{v}\left(\mathrm{p}_{2}, \mathrm{t}_{2}\right)=\mathrm{v}^{\prime}{ }_{2}$. Supongamos, además, que previamente hemos determinado la masa $m\left(p_{1}\right)=m_{1}$ por medio de una balanza (es decir, "importando" dicha masa de un modelo de la mecánica del sólido rígido). Lo único que no presuponemos en nuestro experimento es la masa de p2. $\mathrm{Al}$ contrario, al admitir que $\left\{\mathrm{p}_{1}, \mathrm{p}_{2}\right\}$ junto con sus velocidades y masas constituye un modelo de la mecánica del choque y por tanto satisface la ley de la conservación del momento, podemos escribir

$$
\mathrm{m}_{1} \mathrm{v}_{1}+\mathrm{m}_{2} \mathrm{v}_{2}=\mathrm{m}_{1} \mathrm{v}_{1}^{\prime}+\mathrm{m}_{2} \mathrm{v}_{2}^{\prime},
$$

de donde "despejamos la incógnita" $\mathrm{m}_{2}$ :

$$
\mathrm{m}_{2}=\frac{\mathrm{m}_{1}{ }^{*}\left(\mathrm{v}_{1}^{\prime}-\mathrm{v}_{1}\right)}{\mathrm{v}_{2}-\mathrm{v}_{2}^{\prime}} .
$$

Todo ello no es ninguna novedad. Ahora bien, supongamos que, una vez examinado el choque entre $p_{1}$ y $p_{2}$, e inferida la masa $\mathrm{m}\left(\mathrm{p}_{2}\right)$, utilizamos la misma partícula $\mathrm{p}_{2}$ en un 
choque con otra partícula $\mathrm{p}_{3}$, cuya masa será ahora la incógnita que queremos despejar aplicando la ley de conservación del momento. Ello significa que ahora consideramos un nuevo modelo de la mecánica del choque constituido por $\left\{p_{2}, p_{3}\right\}$ y sus respectivas velocidades y masas. La masa que ahora presuponemos en nuestro cálculo es la de pz, pero ésta no nos la ha proporcionado otra teoría, sino la misma MCH —sólo que un modelo distinto de ella. Para que esta inferencia sea válida, sin embargo, hacemos la asunción implícita de que la masa de $\mathrm{p}_{2}$ no ha cambiado al pasar del primer modelo al segundo. Que ello sea así no se infiere de todo lo que sabemos hasta ahora de la mecánica del choque. Podría ser que, al chocar p2 con p1 tuviera cierta masa, pero al chocar con $p_{3} \neq p_{1}$ su masa cambiara. Que el mundo no posea esta complicación adicional hay que agradecerlo a un axioma implícito de MCH: la masa de una misma partícula no varía cuando ella aparece en dos modelos distintos. Mutatis mutandis vale para la velocidad. En efecto, supongamos que examinamos el choque de $p_{1}$ con $p_{2}$ en el instante t 1 en el marco de un modelo que sólo considera las partículas $\mathrm{p}_{1}$ y $\mathrm{p}_{2}$, y que examinamos el mismo choque en el mismo instante en el marco de un modelo más amplio que, además de $\mathrm{p}_{1}$ y $\mathrm{p}_{2}$, está constituido por otras partículas. Asumimos en tal caso que $\mathrm{v}\left(\mathrm{p}_{1}, \mathrm{t}_{\mathrm{z}}\right)$ dará el mismo valor independientemente de cuál sea el punto de vista ( = modelo considerado). Nuevamente, hay que advertir que el mundo podría ser de otra manera: la presencia o ausencia de varias partículas además de las chocantes podría influir en la velocidad de éstas. Afirmar que no es así, es otro axioma de MCH. Dicho de una manera más abstracta y general: en la mecánica del choque se asume que el valor de una función para un mismo argumento es invariable con respecto al modelo considerado. Muchas teorías empíricas contienen 
condiciones de este tipo, pero no todas. ( $\mathrm{La}$ teoría de las decisiones es una notable excepción.) ${ }^{10}$

Al afirmar la invariancia de velocidad y masa con respecto a los modelos estamos poniendo en relación modelos distintos de la misma teoría. Podríamos decir que se trata de condiciones que ligan varios modelos. Los modelos ligados no van necesariamente por pares —como podría pensarse por los ejemplos anteriores. Hay otros axiomas de este tipo que ligan tres (o más) modelos a la vez. Veamos un ejemplo procedente de la misma $\mathrm{MCH}$.

Supongamos que en un choque (= un modelo) aparece la partícula $\mathrm{p}_{1}$ con masa $\mathrm{m}\left(\mathrm{p}_{1}\right)$; y en otro choque sin relación con el primero aparece la partícula $\mathrm{p}_{2}$ con masa $m\left(\mathrm{p}_{2}\right)$. $\mathrm{Y}$ tomemos ahora las partículas $\mathrm{p}_{1}$ y $\mathrm{p}_{2}$, hagámoslas chocar en un choque totalmente inelástico (más adelante se precisará esta condición), del cual saldrán por así decir "pegadas", componiendo una única partícula, a la que simbolizaremos por ' $\mathrm{p}_{1} \oint \mathrm{p}_{2}{ }^{11}{ }^{11} \mathrm{Si}$ ahora hacemos chocar esta partícula compuesta con otra cualquiera, digamos $\mathrm{p}_{3}$, y necesitamos para nuestros cálculos saber cuál es la masa de $\mathrm{p}_{1} \oint \mathrm{p}_{2}$, asu-

10 Ejemplos de teorías físico-químicas ya reconstruidas con axiomas parecidos a los que acabamos de indicar para MCH son: la geometría física, la mecánica newtoniana de partículas, la mecánica del sólido rígido, la termodinámica gibbsiana del equilibrio, la hidrodinámica clásica y la estequiometría daltoniana. La teoría de las decisiones no cumple este tipo de condiciones porque sus dos funciones, probabilidad subjetiva y utilidad, no son invariantes con respecto al modelo considerado, es decir, con respecto al contexto general en que se coloque al sujeto.

$11 \mathrm{La}$ siguiente observación va destinada a los amantes del rigor absoluto: $\S$ puede introducirse formalmente como una operación algebraica sobre la clase de los dominios de partículas:

$$
\begin{aligned}
& \S: \quad\left(U \pi_{1} x\right)^{2} \longrightarrow U \pi_{1 x} \\
& x \in M_{p}[M C H] \quad x \in M_{p}[M C H]
\end{aligned}
$$

tal que $\&$ es conmutativa, asociativa y no-idempotente. 
miremos que ella es sencillamente

$$
m\left(p_{1} \oint p_{2}\right)=m\left(p_{1}\right)+m\left(p_{2}\right) .
$$

Asumir esto, una vez más, no es ninguna tautología; podría ser que, al "pegarse" ambas partículas por efecto del choque anterior, se perdiera algo de masa. (De hecho, así es efectivamente según la física relativista.) Esta asunción es un nuevo axioma de MCH que expresa, esta vez, una conexión entre tres modelos: aquel en que aparecía $p_{1}$, aquel en que aparecía pr y aquel en que aparece $p_{1} \oint p_{2}$.

Este tipo de axiomas, a los que denominaremos 'condiciones de ligadura', o simplemente 'ligaduras', son completamente triviales si se consideran aplicados a un solo modelo, como es fácil comprender; su chiste estriba justamente en ligar modelos distintos. Por lo tanto, carecería de sentido añadirlos a la lista de condiciones de definición del predicado conjuntista de la teoría (por ejemplo, MCH). En consecuencia, no pueden considerarse ni tipificaciones, ni caracterizaciones, ni leyes sinópticas. ${ }^{12}$ Tampoco expresa vínculos interteóricos, puesto que no ligan modelos de teorías distintas, sino los de una misma teoría; son "intermodélicos", no interteóricos. Son simplemente una nueva categoría de axiomas que hay que identificar en nuestro análisis de las teorías empíricas. Definámoslos.

Def. 8: Una fórmula $A\left(t, \mathrm{x}_{1}, \ldots, \mathrm{x}_{\mathrm{n}}\right)$ es una condición de ligadura de la teoría $T$ si y sólo si existe $\mathrm{j} \in / \mathrm{N}$ tal que $\forall \mathrm{xi}\left(1 \leqslant \mathrm{i} \leq \mathrm{n}_{\mathrm{Xi}} \in \mathrm{Mr}_{\mathrm{P}}[T] \& \pi_{\mathrm{j}} \mathrm{Xi}_{\mathrm{i}}=t\right) .^{13}$

Podríamos generalizar la noción de condición de ligadura

12 Intuitivamente, a lo que más "se parecen" es a las características, pero por las razones apuntadas, no pueden reducirse a tales.

${ }^{13}$ Es más general, sin ser causa de perjuicio, tomar aquí $M_{P}[T]$ en vez de sólo $M[T]$. 
tomando todos los grupos de modelos $\mathrm{x}_{1}, \ldots, \mathrm{x}_{\mathrm{n}}$ (por ejemplo, todos los pares o todas las tríadas, en los ejemplos examinados) que satisfacen el mismo tipo de condición de ligadura y reuniéndolos en una única colección $\mathrm{Z} \subseteq \mathcal{P}\left(\mathrm{M}_{\mathrm{P}}[T]\right)$, cuyos elementos serían justamente los grupos de modelos ligados. Entonces podríamos reformular la definición de condición de ligadura así:

Def. $8^{\prime}$ : Dado $\mathrm{Z} \subseteq \mathcal{P}\left(\mathrm{M}_{\mathrm{r}}[T]\right)$, una fórmula $A(t, \mathrm{Z})$ es una condición de ligadura de la teoría $T$ si y sólo si existe $\mathrm{j} \in / \mathrm{N}$ tal que, para cada $\mathrm{g} \in \mathrm{Z}$, existe una fórmula $A_{\mathrm{g}}(t, \mathrm{~g})$ tal que: $\forall \mathrm{xi}\left(\mathrm{xi}_{\mathrm{i}} \in \mathrm{g} \rightarrow \pi_{\mathrm{j} \mathrm{x} 1}=t\right)$.

Esta última definición, aunque más complicada que la anterior, es preferible porque en ella no hay que casarse con un número determinado $\mathrm{n}$ de modelos ligados por la condición de ligadura.

$\mathrm{Ya}_{\mathrm{a}}$ hemos insinuado antes que, aunque muy frecuentes en las disciplinas empíricas, las condiciones de ligadura no son omnipresentes, ni tienen por qué serlo. (Hay, al parecer, teorías empíricas que se contentan con examinar, por así decir, modelo por modelo, sin tomar un punto de vista más "holista".) Por ello, la tesis axiomatológica que asociamos con el nuevo tipo de axioma será más cauta que las anteriores.

Quinta tesis axiomatológica:

Entre los axiomas de muchas teorías empiricas figura al menos una condición de ligadura.

¿Cuáles serían éstas exactamente en nuestro ejemplo de MCH? Es fácil formularlas después de lo dicho.

$$
(L-\mathrm{I}) \quad \forall \mathrm{g} \in \mathrm{Z} \subseteq \mathrm{M}_{\mathrm{P}}[\mathrm{MCH}] \forall \mathrm{x}, \mathrm{x}^{\prime} \in \mathrm{g} \forall \mathrm{p} \in \pi_{1} \mathrm{x}
$$




$$
\begin{aligned}
: & \forall \mathrm{p}^{\prime} \in \pi_{1} \mathrm{x}^{\prime} \forall \mathrm{t} \in \pi_{3} \mathrm{x} \forall \mathrm{t}^{\prime} \in \pi_{3} \mathrm{x}^{\prime}\left(\mathrm{p}=\mathrm{p}^{\prime} \& \mathrm{t}=\mathrm{t}^{\prime} \rightarrow\right. \\
& \left.\rightarrow \mathrm{c}^{\circ} \mathrm{v}(\mathrm{p}, \mathrm{t})=\mathrm{c}^{\circ} \mathrm{v}\left(\mathrm{p}^{\prime}, \mathrm{t}^{\prime}\right)\right) \\
(L-\mathrm{II}) & \forall \mathrm{g} \in \mathrm{Z} \subseteq \mathrm{M}_{\mathrm{p}}[\mathrm{MCH}] \forall \mathrm{x}, \mathrm{x}^{\prime} \in \mathrm{g} \forall \mathrm{p} \in \pi_{1 \mathrm{x}} \\
& \forall \mathrm{p}^{\prime} \in \pi_{1} \mathrm{x}^{\prime}\left(\mathrm{p}=\mathrm{p}^{\prime} \rightarrow \mathrm{m}(\mathrm{p})=\mathrm{m}\left(\mathrm{p}^{\prime}\right)\right) \\
(L-\mathrm{III}) & \forall \mathrm{g} \in \mathrm{Z} \subseteq \mathrm{M}_{\mathrm{r}}[\mathrm{MCH}] \forall \mathrm{x}, \mathrm{x}^{\prime}, \mathrm{x}^{\prime \prime} \in \mathrm{g} \forall \mathrm{p} \in \pi_{1} \mathrm{x} \\
& \forall \mathrm{p}^{\prime} \in \pi_{1} \mathrm{x}^{\prime} \forall \mathrm{p}^{\prime \prime} \in \pi_{1} \mathrm{x}^{\prime \prime}\left(\mathrm{p}^{\prime \prime}=\mathrm{p} \delta \mathrm{p}^{\prime} \rightarrow \mathrm{m}\left(\mathrm{p}^{\prime \prime}\right)=\right. \\
& \left.=\mathrm{m}(\mathrm{p})+\left(\mathrm{p}^{\prime}\right)\right)
\end{aligned}
$$

¿Hemos concluido nuestra inspección de posibles tipos de axiomas en un teoría empírica? A pesar de la eventual desesperación en la que mi respuesta suma al lector, debo contestar: no. El mismo lector, por muy desesperado que ya esté, deberá admitir, si recuerda sus estudios de física, por elementales que sean, que cuando se expone la mecánica del choque suele hablarse de las leyes del choque, en plural; a lo cual debe seguir la indicación de que, si bien en toda clase de choque vale la ley de conservación del momento, hay casos especiales de choques, los llamados 'elásticos', en los que, además de la susodicha ley, es válida la ley de la conservación de la energía cinética. En nuestro formalismo, esta ley especial se expresaria así:

$$
\left.(E-\mathrm{I}) \sum_{\mathrm{p} \in \mathrm{P}} \mathrm{m}(\mathrm{p}) \% \mathrm{c}^{\circ} \mathrm{v} \mathrm{p}, \mathrm{t}_{1}\right) /^{\mathrm{n}}=\sum_{\mathrm{p} \in \mathrm{P}} \mathrm{m}(\mathrm{p}) \% \mathrm{c}^{\circ} \mathrm{v}\left(\mathrm{p}, \mathrm{t}_{2}\right) /^{2}
$$

“Por qué diablos" — se preguntará el agotado lector"no se introdujo este enunciado, que sin duda tiene el carácter de una ley sinóptica, entre las condiciones de definición de MCH y hubiéramos acabado ya con esta historia de nunca acabar?" La respuesta, amable lector, es que no lo hicimos porque con ello no hubiéramos caracterizado todos los modelos de la teoría que queremos identificar, sino sólo los de una porción de ella: justamente la porción que trata de choques elásticos. Y nosotros pretendíamos identificar la teoria 
entera. La ley de la conservación de la energía cinética, a diferencia de la conservación del momento, constituye sólo una parte de la mecánica del choque - si bien, ciertamente, una parte importante, porque para los choques en los que ella es válida nos proporciona una valiosa información adicional. No hay pues más remedio que establecer una nueva distinción entre aquellos axiomas que son válidos en todos los modelos de una teoría y aquellos que lo son solamente en una subclase, por importante que sea, de los modelos de dicha teoría. Es decir, hay uno(s) axioma(s) de la mecánica del choque que, añadido(s) a las tipificaciones y caracterizaciones, determina(n) $\mathrm{M}[\mathrm{MCH}]$ y hay otro(s) que, añadido(s) a los anteriores, determina(n) alguna subclase $\mathrm{Mi}_{\mathrm{i}}[\mathrm{MCH}] \subseteq \mathrm{M}[\mathrm{MCH}]$. A los primeros los llamamos 'leyes fundamentales' y a los segundos, 'leyes especiales'. ¿Cuál es la forma lógica general de estas leyes especiales? No hay respuesta univoca a esta pregunta. En algunos casos, como el ejemplo que acabamos de ver, se trata también de leyes sinópticas. Pero no es éste siempre el caso. En la mecánica del choque misma se analizan también los llamados 'choques completamente inelásticos'; son aquellos en los que, además de la conservación del momento, se cumple la condición

$(E$-II $) \forall \mathrm{p}, \mathrm{p}^{\prime} \in \mathrm{P}\left(\mathrm{c}^{\circ} \mathrm{v}\left(\mathrm{p}, \mathrm{t}_{2}\right)=\mathrm{c}^{\circ} \mathrm{v}\left(\mathrm{p}^{\prime}, \mathrm{t}_{2}\right)\right)$;

es decir, la velocidad de todas las partículas después del choque es idéntica (todas corren al unísono).

La forma de ( $E$-II) sería "casi" la de una caracterización, si no fuera porque, en rigor, debenios tomar la velo. cidad como la composición $\mathrm{c}^{\circ} \mathrm{v}$ y no sólo como v. En cualquier caso, entre las leyes especiales de una teoría pueden detectarse todo tipo de formas, desde humildes caracterizaciones hasta impresionantes sinopsis. ${ }^{14}$ Lo único que podemos

14 La fauna variopinta de leyes especiales que se manifiesta en teorías más 
decir de general sobre las leyes especiales es lo siguiente:

Def. 9: Una fórmula $A$ es una ley especial de la teoría $T$ sólo si existe $\mathrm{x} \in \mathrm{M}[T]$ tal que $\mathrm{x}$ satisface $A$ y existe $\mathrm{x}^{\prime} \in \mathrm{M}[T]$ tal que $\mathrm{x}^{\prime}$ no satisface $A$.

A esta semi-definición asociamos, con la cautela de la que hicimos gala en el paso anterior, la siguiente (¡y última!)

Sexta tesis axiomatológica:

Entre los axiomas de muchas teorías empíricas existe al menos una ley especial.

En el caso de MCH, $(E$-I) y (E-II) son sus dos leyes especiales más significativas. Pero podríamos añadir más, si fuera conveniente. El conjunto de leyes especiales de una teoría empírica es, por su naturaleza misma, potencialmente abierto. Este carácter abierto del conjunto de leyes especiales es más notorio en teorías de estructura más compleja que MCH, como la mecánica newtoniana y la termodinámica del equilibrio. Ello tiene que ver con la forma particular de la ley fundamental. ${ }^{15}$ Pero éste es un aspecto de ciertas teorías que no he podido apresar axiomáticamente y en el que, en consecuencia, no quiero entrar aquí. El presente estudio sólo pretendía detectar aquellas categorías constituyentes de una teoría empírica que pueden determinarse axiomáticamente. Ellas no agotan todo lo que puede averiguarse acerca de la identidad de una teoría. No obstante, su diversidad y complejidad es suficientmente notoria como para que merezcan nuestra atención filosófica.

complejas que $\mathrm{MCH}$ podrá contemplarse examinando la forma lógica de las múltiples leyes especiales que aparecen en la reconstrucción de la mecánica newtoniana de partículas y la termodinámica simple del equilibrio presentada en Moulines, Exploraciones metacientíficas, op. cit., cap. 2.5 y 2.6 .

${ }_{15}$ Cf. Moulines, op. cit., cap. 2.3 . 


\section{SUMMARY}

The author analyses, within a framework of the structuralist conception of science, different types of axioms that may occurr in empirical theories, set-theoretically axiomatized; in the process, he states six theses, offering formal definitions to characterize each type of axiom, except for the last one. The following set-theoretical axiomatization of classical collision mechanics $(\mathrm{CM})$ is used as illustration:

$C M(x)$ iff there exists $P, S, T, c, v, m, t_{1}, t_{2}$ such that:

(0) $\mathrm{x}=\langle\mathrm{P}, \mathrm{S}, \mathrm{T}, / \mathrm{R}, \mathrm{c}, \mathrm{v}, \mathrm{m}\rangle$

(1) $P$ is a finite non-empty set

(2) $\mathrm{S}$ is a non-empty set

(3) $\mathrm{T}=\left\{\mathrm{t}_{1}, \mathrm{t}_{2}\right\}$

(4) $c$ maps $S$ onto $/ \mathrm{R}^{3}$

(5) $v$ maps $P \times T$ into $S$

(6) $m$ is a function from $P$ into $/ R$ such that for all $p \in P$, $m(p)>0$

(7) $\sum_{p \in P} m(p) \cdot c^{\circ} v\left(p, t_{1}\right)=$ $\sum_{p \in P} m(p) \cdot c^{\circ} v\left(p, t_{2}\right)$

(where '/R' designs the real numbers and ' $O$ ' the composition of functions). This axiomatization is completed with a set of informal statements that offers the intuitive physical interpretation of the basic concepts; e.g., "The elements of P are particles" and " $\mathrm{m}$ is the mass of each particle". First thesis: Among the axioms of an empirical theory at least one typification occurs. Through the "constructive echelons" of the projection $(\pi)$, power set $(\mathcal{P})$ and cartesian product $(X)$ operations, the typifications make explicit and precise the type of the set-theoretical entities that corresponds to some of the basic concepts: relations and functions. $E$.g., for $m$, we have: $m \in P\left(\pi_{1}\right.$ $\left.\left.\langle\mathrm{P}, / \mathrm{R}\rangle \times \pi_{2}<\mathrm{P}, / \mathrm{R}\right\rangle\right)$. The typifications have no empirical meaning at all; they can be stated by means of pure conceptual analysis.

Second thesis: Among the axioms of an empirical theory at least one characterization occurs. Obviously, the typifications do not exhaust all that can be said in set-theoretical terms about the basic concepts. 
The characterizations supply additional information which contains, although in a minimal degree, certain factual meaning, as we can see in the characterization of $\mathrm{m}: \mathrm{Func}(\mathrm{m}) \& \forall p \in P(\mathrm{~m}(\mathrm{p})>0)$, where 'Func' is a term for the general concept of function.

Third thesis: Among the axioms of an empirical theory al least one sinoptic law occurs. What type of axiom is (7), the momentum conservation law? Evidenttly, it is neither a typification nor a characterization; it is just a scientific law. Laws are the kind of essential statements in a theory (without a law a theory is not a theory); they contain the maximum amount and most important empirical meaning of a theory among all the axioms.

It would seem that the three types of axioms listed are sufficient to describe an axiomatization of an empirical theory. However, there are other components that contribute to the identification, which are necessary for the empirical applications of theories.

Fourth thesis: Among the axioms of an empirical theory at least one interpretation axiom occurs. An interpretation axiom is a statement that asserts a link between the potential models of a theory and the (actual) models of another. These links, as inter-theoretic relations, are vital for the empirical applications of theories; without them we do not know, $e$. g., to what kind of things the concepts of mass or speed are applicable. In CM we need interpretation axioms for the existing links between itself and, e.g., the chassical kinematics, (physical) euclidean geometry and chronometry.

Fifth thesis: Among the axioms of many empirical theories at least one constraint occurs. This type of axioms states a relation among models of the same theory such that it allows to export data of a model in order to make e.g., a calculation or a measurement for a further model. In CM we have constraints for the invariation of both mass and speed and for the extensivity of mass.

Sixth thesis: Among the axioms of many empirical theories at least one special law occurs. Is (7) the only law of CM? Certainly not. In some kind of applications of CM, e.g., the conservation of kinematics energy law is used. However, this law does not hold in all applications of CM and, thus, it is not a general law but rather a special one. The same situation occurs in many empirical theories, in particular, in the complexes. But due to the open character of the set of the special laws, they can hardly be captured in a formal fashion.

[J. L. Rolleri] 